ITFA-2004-17

hep-th/0404176

\title{
AdS/CFT correspondence and Geometry
}

\author{
Ioannis Papadimitriou ${ }^{1}$ and Kostas Skenderis ${ }^{2}$ \\ Institute for Theoretical Physics, University of Amsterdam, \\ Valkenierstraat 65, 1018 XE Amsterdam, The Netherlands.
}

\begin{abstract}
In the first part of this paper we provide a short introduction to the AdS/CFT correspondence and to holographic renormalization. We discuss how QFT correlation functions, Ward identities and anomalies are encoded in the bulk geometry. In the second part we develop a Hamiltonian approach to the method of holographic renormalization, with the radial coordinate playing the role of time. In this approach regularized correlation functions are related to canonical momenta and the near-boundary expansions of the standard approach are replaced by covariant expansions where the various terms are organized according to their dilatation weight. This leads to universal expressions for counterterms and one-point functions (in the presence of sources) that are valid in all dimensions. The new approach combines optimally elements from all previous methods and supersedes them in efficiency.
\end{abstract}

\footnotetext{
${ }^{1}$ ipapadim@science.uva.nl

${ }^{2}$ skenderi@science.uva.nl
} 


\section{Contents}

$\begin{array}{lll}1 & \text { Introduction } & 2\end{array}$

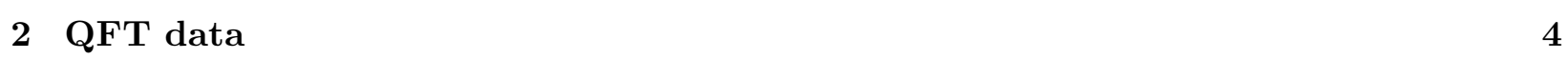

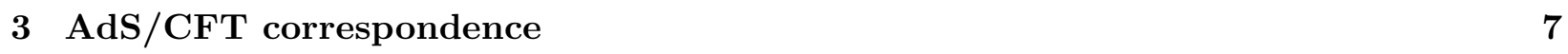

4 Hamiltonian approach to Holographic Renormalization $\quad 11$

4.1 Pure gravitv case . . . . . . . . . . . . . . . . . . . . . . 19

4.2 Gravity coupled to scalars . . . . . . . . . . . . . . . . . . 24

$\begin{array}{lll}5 & \text { Conclusions } & 27\end{array}$

\section{Introduction}

The AdS/CFT correspondence [1, 2, 3] (for reviews see 4, 5]) relates string theory on (locally asymptotically) AdS spacetimes (times a compact space) with a quantum field theory (QFT) "residing" on the conformal boundary of the bulk spacetime. ${ }^{3}$ In a specific limit, which is a strong coupling limit of the boundary theory, the bulk theory reduces to classical gravity coupled to certain matter fields. In this limit QFT data is encoded in classical geometry. The aim of this contribution is to discuss how to extract this data from the bulk geometry.

In the first part of this paper we give a brief review of the AdS/CFT correspondence and of holographic renormalization for non-experts. We start our discussion from the QFT side by reviewing what QFT data we would like to obtain from gravity. This data consists of correlation functions of gauge invariant operators and of symmetry relations. Symmetries of the QFT action imply relations among correlation functions, the so-called Ward identities. Such relations are "kinematical" and can be established without the need to actually compute the correlation functions. Sometimes, however, quantum effects imply that some of the classical symmetries are broken at the quantum level: the corresponding Ward identities are anomalous. The symmetries of the quantum theory are thus encoded in the corresponding Ward identities and anomalies.

The next task is to describe how to obtain correlation functions, Ward identities and anomalies using the AdS/CFT correspondence. We outline the prescription of [2, 3] for the computation of correlation functions and discuss how to deal with the infinities arising in such computations. This is dealt with via the formalism of holographic renormalization [6, 7, 8], (for a review see [9]; for

\footnotetext{
${ }^{3}$ In the first examples discussed in the literature, the bulk spacetime was exactly AdS (times a compact space) and the dual theory was a conformal field theory (CFT). This motivated the name "AdS/CFT correspondence". Our discussion is applicable under the more general circumstances mentioned above.
} 
related work see [10, 11] - a more complete list of references can be found in the review). This formalism automatically incorporates the "kinematical" constraints, i.e. the Ward identities and their anomalies, and identifies the part of the geometry where the "dynamical" information, i.e. the correlation functions, is encoded. A central role in this method is played by the fact that one can perturbatively work out the asymptotics of all bulk fields to sufficiently high order using the radial distance from the boundary of AdS as a small parameter ${ }^{4}$ [12] (for relevant math reviews see [13, 14]). Correlation functions are encoded in specific coefficients in the asymptotic expansion of the bulk fields and Ward identities and anomalies originate from certain relations that these coefficients satisfy.

This method, even though complete, is not very efficient as we discuss later. The last part of the paper is devoted to developing a "Hamiltonian" version of the method, where the radial coordinate plays the role of time. In this approach the focus is shifted from the on-shell supergravity action to the canonical momenta of the bulk fields. The latter are associated with (regularized) correlation functions of gauge invariant operators [15]. To obtain renormalized correlation functions we need to subtract the infinities. This was done in the standard approach via the near-boundary analysis. In the new approach we use instead the fact that there is a well defined dilatation operator. This allows us to develop a covariant expansion of the asymptotic solution where the various terms are organized according to their dilatation eigenvalue. This leads to a faster algorithm for determining counterterms and correlation functions. ${ }^{5}$ In particular, we obtain universal recursion relations for the asymptotic solutions and counterterms that are valid in all dimensions.

This paper is organized as follows. In the next section we discuss the QFT data that enters in the discussion of the AdS/CFT correspondence. The discussion is illustrated by a number of simple examples and assumes only a general familiarity with QFT. In section 3 we present a brief review of the AdS/CFT correspondence and of holographic renormalization. More details can be found in the reviews listed above. Sections 2 and 3 are aimed at non-experts that want to get a flavor of the ideas and techniques involved in the AdS/CFT correspondence. Experts can safely move directly to section 4 where the new Hamiltonian formulation is discussed systematically. This section is self-contained and can be read independently of the previous sections.

Throughout this paper we work with Euclidean signature. All results, however, can be straightforwardly continued to any other signature.

\footnotetext{
${ }^{4}$ From the point of view of the dual field theory we expand around a UV fixed point, the small parameter being the inverse energy.

${ }^{5} \mathrm{~A}$ different Hamiltonian approach to renormalization using the Hamilton-Jacobi equation [15] was developed in [16], see also 17] and references therein.
} 


\section{QFT data}

We discuss in this section the quantum field theory data that we would like to extract from the bulk geometry. We would say that a QFT is solved if we determine all correlation functions of all gauge invariant operators. The set of gauge invariant operators depends on the theory under consideration. Examples of such operators are the stress energy tensor $T_{i j}$, currents $J^{i}$ associated with global symmetries and scalar operators $O$. As mentioned in the introduction, when the bulk spacetime is exactly $A d S$ (times a compact space) the dual quantum field theory is a conformal field theory (CFT). The discussion in this section will refer to CFTs, but all considerations have a straightforward generalization to QFTs that can be viewed either as deformations of the CFTs by relevant or marginal operators or to CFTs with spontaneously broken conformal invariance.

The examples of AdS/CFT correspondence involve specific CFTs - the most studied case being the maximally supersymmetric gauge theory in four dimensions, the $\mathcal{N}=4 \mathrm{SYM}$ theory. The discussion below is focused on general properties that do not depend on the details of the specific CFT. Given a (perturbative) CFT specified by set of fields $\varphi^{A}$ one can work out the set of gauge invariant composite operators $\mathcal{O}\left(\varphi^{A}\right)$. Their correlation functions can then be computed in perturbation theory. To give an elementary example of a CFT correlator consider a scalar operator $O_{\Delta}$ of conformal weight $\Delta$. In this case the form of the 2 -point function is fixed by conformal invariance,

$$
\left\langle O_{\Delta}(x) O_{\Delta}(0)\right\rangle=\frac{c(g, \Delta)}{x^{2 \Delta}},
$$

where $c(g, \Delta)$ is a constant that depends on the coupling constant of the theory $g$ and the conformal dimension $\Delta$ of the operator. One may set it to one by a choice of normalization of $O_{\Delta}$ but we shall not do so. Our objective is to understand how to extract this and more complicated higher point functions (that are not determined by symmetries) from the bulk geometry.

In general, symmetries of the classical action imply relations among correlation functions, the so-called Ward identities. To give an example: Poincaré invariance of the classical action implies (classically) that the stress energy tensor is conserved,

$$
\partial^{i} T_{i j}=0
$$

At the quantum level this implies relations among certain correlation functions. For instance,

$$
\partial_{x}^{i}\left\langle T_{i j}(x) O(y) O(z)\right\rangle=\partial_{j} \delta(x-y)\langle O(x) O(z)\rangle+\partial_{j} \delta(x-z)\langle O(y) O(x)\rangle .
$$

Some of the classical symmetries, however, are broken by quantum effects. For example, the stress energy tensor of a field theory that is classically conformally invariant is traceless, but quantum effects may break this symmetry

$$
T_{i}^{i}=0 \quad \text { classical, } \quad\left\langle T_{i}^{i}\right\rangle=\mathcal{A} \text { quantum }
$$


Since $T_{i}^{i}$ generates scale transformations, the conformal anomaly captures the fact that the correlators, even though they are CFT correlators, are not scale invariant,

$$
\mu \frac{\delta}{\delta \mu}\left\langle O_{1}\left(x_{1}\right) \cdots O_{n}\left(x_{n}\right)\right\rangle=A \delta\left(x_{1}, \ldots, x_{n}\right)
$$

where $\delta\left(x_{1}, \ldots, x_{n}\right)=\delta\left(x_{1}-x_{2}\right) \delta\left(x_{2}-x_{3}\right) \ldots \delta\left(x_{n-1}-x_{n}\right)$, and $A$ is related to $\mathcal{A}$ in a way we specify below. Notice that the violation of conformal invariance is a contact term. In a general QFT (i.e. not conformal) (5) is replaced by the beta function equation.

To understand how an anomaly can arise consider the 2-point function in (11). The form of this correlator (for $x^{2} \neq 0$ ) is completely fixed (up to normalization) by conformal invariance. Depending on the conformal dimension, however, the correlator may suffer from short distance singularities. Consider the case $\Delta \sim d / 2+k$, where $d$ is the spacetime dimension and $k$ is an integer. As $x^{2} \rightarrow 0$ the correlator behaves as

$$
\frac{1}{x^{2 \Delta}} \sim \frac{1}{d+2(k-\Delta)} \frac{\Gamma(d / 2)}{2^{2 k} k ! \Gamma(d / 2+k)} S^{d-1} \square^{k} \delta^{(d)}(x)
$$

where $S^{d-1}=2 \pi^{d / 2} / \Gamma(d / 2)$ is the volume of the unit $(d-1)$-sphere and $\square=\delta^{i j} \partial_{i} \partial_{j}$. We thus find that there is a pole at $\Delta=d / 2+k$. To produce a well-defined distribution we use dimensional regularization and subtract the pole. For concreteness we consider the case $k=0$ (all other cases follow by differentiation w.r.t. to $x$, see [18]). Minimal subtraction yields [19, 18]

$$
\begin{aligned}
\left\langle O_{d / 2}(x) O_{d / 2}(0)\right\rangle_{R} & =c(g, d / 2) \lim _{\Delta \rightarrow d / 2}\left(\frac{1}{x^{2 \Delta}}-\frac{\mu^{2 \Delta-d}}{d-2 \Delta} S^{d-1} \delta^{(d)}(x)\right) \\
& =-c(g, d / 2) \frac{1}{2(d-2)} \square \frac{1}{\left(x^{2}\right)^{\frac{1}{2} d-1}}\left(\log \mu^{2} x^{2}+\frac{2}{d-2}\right) .
\end{aligned}
$$

where the subscript $R$ indicates that this is a renormalized correlator. The scale $\mu$ is introduced, as usual in dimensional regularization, on dimensional grounds. The renormalized correlator agrees with the bare one away from coincident points but is also well-defined at $x^{2}=0$. Let us now consider the scale dependence of the renormalized correlator,

$$
\mu \frac{\partial}{\partial \mu}\left\langle O_{d / 2}(x) O_{d / 2}(0)\right\rangle_{R}=S^{d-1} c(g, d / 2) \delta^{(d)}(x) .
$$

where we used $\square\left(x^{2}\right)^{-d / 2+1}=-(d-2) S^{d-1} \delta^{(d)}(x)$. Thus the renormalized correlation function exhibits a violation of scale invariance. We shall soon connect this to the violation of the tracelessness condition of the stress energy tensor.

Recall that correlation function of composite operators may be computed by introducing sources that couple to them. The generating functional of correlation functions then has the following path integral representation,

$$
Z\left[g_{(0)}, \phi_{(0)}\right]=\int\left[D \varphi^{A}\right] \exp \left(-\int d^{d} x \sqrt{g_{(0)}}\left[\mathcal{L}_{C F T}\left(\varphi^{A} ; g_{(0)}\right)+\phi_{(0)} O\left(\varphi^{A}\right)\right]\right)
$$


where $\varphi^{A}$ represents collectively all fields of the theory, $g_{(0)}$ is a background metric (which serves as a source for the stress energy tensor), $\mathcal{L}_{C F T}$ is the Lagrangian density for the CFT and $\phi_{(0)}$ is a source for the operator $O$. Correlation functions can now be computed by differentiating w.r.t. sources and then setting the sources to zero. For instance, the connected two-point function of $O$ on flat spacetime $\left(g_{(0) i j}=\delta_{i j}\right)$ is given by

$$
\langle O(x) O(0)\rangle=\left.\frac{\delta^{2} W}{\delta \phi_{(0)}(x) \delta \phi_{(0)}(0)}\right|_{\phi_{(0)}=0}
$$

where $W=\log Z$ is the generating functional of connected correlators. Given a Lagrangian density $\mathcal{L}_{Q F T}$ one could thus compute the correlation functions of $O$ by first (perturbatively) computing $Z\left[g_{(0)}, \phi_{(0)}\right]$. Such computations however are plagued by infinities and to make sense of them one needs to renormalize the theory. To subtract the divergences one may add counterterms to the action. If the counterterms break a classical symmetry, then this symmetry is anomalous.

A slightly different route is to first compute in general the one-point functions in the presence of sources,

$$
\left\langle T_{i j}(x)\right\rangle_{s}=-\frac{2}{\sqrt{g_{(0)}(x)}} \frac{\delta W\left[g_{(0)}, \phi_{(0)}\right]}{\delta g_{(0)}^{i j}(x)}, \quad\langle O(x)\rangle_{s}=-\frac{1}{\sqrt{g_{(0)}(x)}} \frac{\delta W\left[g_{(0)}, \phi_{(0)}\right]}{\delta \phi_{(0)}(x)}
$$

where the subscript $s$ in the correlation functions indicate that the sources are non-zero. Correlation functions are then computed by further differentiating w.r.t. sources and setting the sources to zero. This reformulation will be proved useful later when we show how to compute correlation functions holographically (i.e. using the AdS/CFT correspondence). Another advantage is that one can express compactly many Ward identities. For instance, invariance of $Z$ under diffeomorphisms,

$$
\delta g_{(0)}^{i j}=-\left(\nabla^{i} \xi^{j}+\nabla^{j} \xi^{i}\right), \quad \delta \phi_{(0)}=\xi^{j} \nabla_{j} \phi_{(0)}
$$

implies

$$
\nabla^{i}\left\langle T_{i j}(x)\right\rangle_{s}=-\langle O(x)\rangle_{s} \nabla_{j} \phi_{(0)}(x)
$$

Differentiating now twice w.r.t. $\phi_{(0)}$ and then setting $\phi_{(0)}=0, g_{(0) i j}=\delta_{i j}$ leads to (3).

Using the fact that the trace of the stress energy tensor is the generator of conformal transformations we arrive at [18

$$
\int d^{d} x \sqrt{g_{(0)}} g_{(0)}^{i j}\left\langle T_{i j}\right\rangle=\sum_{k=1}^{\infty} \frac{(-1)^{k}}{k !} \int \prod_{i=1}^{k}\left(d^{d} x_{i} \sqrt{g_{(0)}} J\left(x_{i}\right)\right) \mu \frac{\partial}{\partial \mu}\left\langle\mathcal{O}\left(x_{1}\right) \cdots \mathcal{O}\left(x_{k}\right)\right\rangle
$$

where $J$ denotes all sources and $\mathcal{O}$ the corresponding operators. In our case, $J=\left\{\phi_{(0)}, g_{(0) i j}\right\}$ and $\mathcal{O}=\left\{O, T_{i j}\right\}$. Clearly, the expectation value of the stress energy tensor is non-vanishing if the scale derivative of the correlator is non-vanishing. In particular, we have seen in (8) that the scale 
derivative of the 2-point function of an operator of dimension $d / 2$ yields a delta function. Inserting this in (14) we obtain

$$
\left\langle T_{i}^{i}\right\rangle=\frac{1}{2} S^{d-1} c(g, d / 2) \phi_{(0)}^{2}
$$

So, in this case, $\mathcal{A}=A \phi_{(0)}^{2} / 2$ and $A=S^{d-1} c(g, d / 2)$. This result generalizes to all operators of dimension $\Delta=d / 2+k$ with result [18]

$$
\left\langle T_{i}^{i}\right\rangle=\frac{1}{2} c_{k} \phi_{(0)} \square^{k} \phi_{(0)}, \quad c_{k}=\frac{\pi^{d / 2}}{2^{2 k-1} \Gamma(k+1) \Gamma(k+d / 2)} c(g, \Delta) .
$$

These considerations were valid for flat spacetime. When the background is curved, the results generalize to

$$
\left\langle T_{i}^{i}\right\rangle=\frac{1}{2} c_{k} \phi_{(0)} P_{k} \phi_{(0)}+\left(a E+\sum_{i} c_{i} W^{i}\right)+\nabla_{i} J^{i}
$$

$P_{k}$ is equal to $\square^{k}$ when the background is flat and transforms covariantly under Weyl transforms $g_{(0)} \rightarrow g_{(0)} e^{2 \sigma}$

$$
P_{k} \rightarrow e^{-(d / 2+k) \sigma} P_{k} e^{(d / 2-k) \sigma}
$$

For instance, for $k=1$,

$$
P_{1}=\square+\frac{d-2}{4(d-1)} R .
$$

The two terms inside the parenthesis in (17) are purely gravitational and are present only when $d$ is even. $E$ is the Euler density, $W^{i}$ is a basis of Weyl invariants of dimension $d$ and $a$ and $c_{i}$ are numerical constants that depend on the field content of the theory. For instance, in $d=4$ there is one Weyl invariant (the square of the Weyl tensor), in $d=6$ there are three such tensors, etc. The last term in (17) is scheme dependent, i.e. it can be modified by local finite counterterm terms in the action. In general there may be additional terms in (17) that depend on higher powers of the sources $\phi_{(0)}$. These would be related to singularities in higher-point functions. The structure of (17) is dictated by the fact that the integrated conformal anomaly is itself conformally invariant 20, 21.

The AdS/CFT duality implies that all this data is encoded in the geometry. We discuss in the next section how to recover them from the bulk geometry.

\section{AdS/CFT correspondence}

The AdS/CFT correspondence states that there is an exact equivalence between string theory on (locally) asymptotically AdS (AAdS) spacetimes (times a compact space) and a quantum field theory that "resides" on the conformal boundary of the AAdS spacetime. In the regime where the one description is perturbative the other one is strongly coupled. We will work in the regime where 
the gravitational description is valid and we will describe how to obtain the QFT data described in the previous section.

The basic AdS/CFT dictionary is as follows:

1. Gauge invariant operators of the boundary theory are in one-to-one correspondence with bulk fields. For example, the bulk metric corresponds to the stress energy tensor of the boundary theory.

2. The leading boundary behavior of the bulk field is identified with the source of the dual operator.

3. The string partition function (which is a functional of the fields parameterizing the boundary behavior of the bulk fields) is identified with the generating functional of QFT correlation functions.

At low energies and to leading order the AdS/CFT prescription reads,

$$
S_{\text {on-shell }}\left[f_{(0)}\right]=-W\left[f_{(0)}\right]
$$

where $S_{\text {on-shell }}\left[f_{(0)}\right]$ is the on-shell value of the supergravity action, $f_{(0)}$ denotes collectively all fields parameterizing the boundary values of bulk fields and $W$ is the generating functional of connected graphs (see the discussion below (9)). It follows that one can compute correlators of the (strongly coupled) QFT gravitationally by first evaluating the on-shell value of the supergravity action and then differentiating w.r.t. the boundary values, e.g.

$$
\langle O(x) O(0)\rangle=-\left.\frac{\delta^{2} S_{\text {on-shell }}}{\delta \phi_{(0)}(x) \delta \phi_{(0)}(0)}\right|_{\phi_{(0)}=0}
$$

A naive use of these formulas however yields infinite answers - the on-shell value of the action is infinite due to the infinite volume of the AAdS spacetime. The goal of holographic renormalization is to make these formulas well-defined.

The general form of the bulk action is

$$
S=\int d^{d+1} x \sqrt{g}\left[-\frac{1}{2 \kappa^{2}} R+\frac{1}{2} g^{\mu \nu} \partial_{\mu} \Phi \partial_{\nu} \Phi+V(\Phi)+\cdots\right]
$$

where $\kappa^{2}=8 \pi G_{d+1}\left(G_{d+1}\right.$ is Newton's constant) and the dots indicate contribution of additional fields such as gauge fields, fermions, antisymmetric tensors. The analysis below generalizes straightforwardly to include such fields (but it becomes a lot more tedious). Restricting to the gravity-scalar sector means that we only study correlation functions of the stress energy tensor and a scalar operator. The potential has the form,

$$
V(\Phi)=\frac{\Lambda}{\kappa^{2}}+\frac{1}{2} m^{2} \Phi^{2}+g \Phi^{3}+\cdots
$$


where $\Lambda$ is the cosmological constant and the mass $m^{2}$ of the scalar field is related to the dimension $\Delta$ of the dual operator by $m^{2}=(\Delta-d) \Delta$. The bulk field equations are given by

$$
G_{\mu \nu}=\kappa^{2} \tilde{T}_{\mu \nu}(\Phi), \quad \square_{g} \Phi=\partial V / \partial \Phi
$$

where $G_{\mu \nu}$ is the Einstein tensor, $\square_{g} \Phi=\frac{1}{\sqrt{g}} \partial_{\mu}\left(\sqrt{g} g^{\mu \nu} \partial_{\nu} \Phi\right)$ and $\tilde{T}_{\mu \nu}(\Phi)$ is the stress energy tensor associated with the scalar field $\Phi$ (see (34) ).

The method of holographic renormalization now consists of the following steps (a more detailed discussion can be found in [9]).

\section{Asymptotic solutions}

In the first step one works out the most general asymptotic solutions with given Dirichlet data

$$
\begin{aligned}
d s^{2} & =\frac{d \rho^{2}}{4 \rho^{2}}+\frac{1}{\rho} g_{i j}(x, \rho) d x^{i} d x^{j} \\
\Phi(x, \rho) & =\rho^{(d-\Delta) / 2} \phi(x, \rho)
\end{aligned}
$$

where $^{6}$,

$$
\begin{aligned}
g_{i j}(x, \rho) & =g_{(0) i j}+\rho g_{(2) i j}+\cdots+\rho^{d / 2}\left(g_{(d) i j}+\log \rho h_{(d) i j}\right)+\cdots \\
\phi(x, \rho) & =\phi_{(0)}+\rho \phi_{(2)}+\cdots+\rho^{\Delta-d / 2}\left(\phi_{(2 \Delta-d)}+\log \rho \psi_{(2 \Delta-d)}\right)+\cdots
\end{aligned}
$$

In this expansion, $g_{(0) i j}$ and $\phi_{(0)}$ are identified with the QFT sources that couple to the dual operators, as discussed in the previous section.

Inserting these expansions in the bulk field equations (24) one finds that all coefficients but $\phi_{(2 \Delta-d)}$ and the traceless transverse part of $g_{(d) i j}$ are locally determined by $g_{(0) i j}$ and $\phi_{(0)}$ [12, 6, 7 ] (see the appendices of [7] for explicit expressions of the coefficients). The part of $g_{(d) i j}$ that is determined, i.e. $\nabla^{i} g_{(d) i j}$ and $\operatorname{Tr} g_{(d)}$, encodes Ward identities and anomalies, as we discuss below. We will call $g_{(d) i j}$ and $\phi_{(2 \Delta-d)}$ the response functions. The logarithmic terms appear only in special cases: $h_{(d)}$ only in even dimensions and $\psi_{(2 \Delta-d)}$ only when $\Delta-d / 2$ is an integer. Both of them are directly related to the conformal anomalies discussed in the previous section: $h_{(d)}$ is the metric variation of the gravitational part of the conformal anomaly and $\psi_{(2 \Delta-d)}$ is the variation w.r.t. $\phi_{(0)}$ of the matter part of the conformal anomaly [7].

\section{On-shell divergences}

Having obtained the asymptotic solutions we now obtain the most general divergences of the on-shell action,

$$
S_{r e g}\left[g_{(0)}, \phi_{(0)} ; \epsilon\right]=\int d^{d} x \sqrt{g_{(0)}}\left(\sum_{\nu} a_{(\nu)} \epsilon^{-\nu}-\log \epsilon a_{(d)}\right)+\mathcal{O}(\epsilon)
$$

\footnotetext{
${ }^{6}$ In general, the expansions may involve half integral powers of $\rho$. In such cases it is more natural to use a new radial coordinate $r$, where $\rho=r^{2}$.
} 
where $\epsilon$ is a cut-off in the radial coordinate, $\rho \geq \epsilon$. It turns out all coefficients $a_{(\nu)}$ depend only on $g_{(0)}$ and $\phi_{(0)}$ but not on the undetermined coefficients $g_{(d)}$ and $\phi_{(2 \Delta-d)}$. The coefficient $a_{(d)}$ is equal to the conformal anomaly of the dual CFT [6].

\section{Counterterms and renormalized action}

To obtain a well-defined on-shell action we should subtract the infinities and then remove the regulator. To do this we first express the divergent terms found in the previous step in terms of induced fields at the hypersurface $\rho=\epsilon$. This entails inverting the asymptotic series obtained in the first step and inserting it in the divergent terms obtained in the second step. This is one of the most laborious steps of the procedure. The end result is the counterterm action, $S_{c t}$. The renormalized action is defined by

$$
S_{r e n}=\lim _{\epsilon \rightarrow 0} S_{s u b}, \quad S_{s u b}=S_{r e g}+S_{c t} .
$$

\section{1-point functions in the presence of source}

We can now differentiate the renormalized action to obtain the 1-point function in the presence of sources [7],

$$
\begin{aligned}
\left\langle T_{i j}(x)\right\rangle_{s} \equiv \frac{2}{\sqrt{g_{(0)}(x)}} \frac{\delta S_{r e n}}{\delta g_{(0)}^{i j}(x)} & =\lim _{\epsilon \rightarrow 0}\left(\frac{1}{\epsilon^{d / 2-1}} \frac{2}{\sqrt{\gamma(x, \epsilon)}} \frac{\delta S_{s u b}}{\delta \gamma^{i j}(x, \epsilon)}\right) \\
& =\frac{d}{2 \kappa^{2}} g_{(d) i j}+X\left[g_{(0)}, \phi_{(0)}\right] \\
\langle O(x)\rangle_{s} \equiv \frac{1}{\sqrt{g_{(0)}(x)}} \frac{\delta S_{r e n}}{\delta \phi_{(0)}(x)} & =\lim _{\epsilon \rightarrow 0}\left(\frac{1}{\epsilon^{\Delta / 2}} \frac{1}{\sqrt{\gamma(x, \epsilon)}} \frac{\delta S_{s u b}}{\delta \Phi(x, \epsilon)}\right) \\
& =(d-2 \Delta) \phi_{(2 \Delta-d)}+Y\left[g_{(0)}, \phi_{(0)}\right]
\end{aligned}
$$

where $X\left[g_{(0)}, \phi_{(0)}\right]$ and $Y\left[g_{(0)}, \phi_{(0)}\right]$ are (known) local expressions that depend on sources. The first equality is a definition. In the second equality we expressed the 1-point function as a limit of the regulated 1-point function. The regulated 1-point function can be computed in all generality and the limit can be explicitly taken. This is a straightforward but rather tedious computation. The result is the one shown above. We thus find that the correlation functions depend on the coefficient that the asymptotic analysis left undetermined. As discussed above, the near boundary analysis does determine the divergence and trace of $g_{(d) i j}$. This means that the divergence and trace of $\left\langle T_{i j}(x)\right\rangle_{s}$ can be determined. This yields the Ward identities, including anomalies, that we discussed in the previous section. The relations (31) imply that the pairs $\left(g_{(0)}, g_{(d)}\right)$ and $\left(\phi_{(0)}, \phi_{(2 \Delta-d)}\right)$ are conjugate pairs.

\section{Correlation functions}

To obtain higher point functions we should further differentiate (31) w.r.t. the sources. The expressions $X\left[g_{(0)}, \phi_{(0)}\right]$ and $Y\left[g_{(0)}, \phi_{(0)}\right]$ lead to only local contributions. The (non-local) $n$-point 
function is thus encoded in the dependence of $g_{(d)}$ and $\phi_{(2 \Delta-d)}$ on the sources. We thus reach the conclusion:

The theory is solved if we determine the response functions in terms of the sources.

To obtain such a relation we need a regular exact (as opposed to asymptotic) solution of the bulk equations with the boundary conditions specified by the sources. In the absence of more powerful methods one can proceed perturbatively. One can determine the response functions to linear order by solving the bulk field equations linearized around a background solution [8]. (The background solution specifies the vacuum of the dual QFT, see section 6.1 of [9]). Higher-point functions can be computed by solving the bulk equations perturbatively in a bulk coupling constant. Examples have been discussed in 9 , 22].

The procedure described here is general and can be carried out in all cases. The steps however appear to have certain redundancy. In step 1 and 2 the asymptotic solution and divergences are obtained in terms of the Dirichlet data. In order to obtain the counterterms however one should invert the asymptotic series. Then the 1-point functions are obtained in terms of the induced fields at $\rho=\epsilon$ and the asymptotic solution is used again to obtain the final expression for 1-point functions. Clearly, it would be desirable to avoid having to go back and forth from asymptotic data to covariant fields. A related issue is the following. In step 2 we mentioned that the divergences depend only on the sources but not the response functions. This followed from an explicit computation. It would be more satisfying to make this manifest. We discuss in the next section an approach that removes these drawbacks. A related work that also leads to simplifications can be found in [16].

\section{Hamiltonian approach to Holographic Renormalization}

Let $\bar{M}$ be a conformally compact, Riemannian (d+1)-manifold, $M$ its interior and $\partial M$ its boundary. We will consider the following action for the Riemannian metric $g_{\mu \nu}$ on $\bar{M}$

$$
S_{g r}[g]=-\frac{1}{2 \kappa^{2}}\left[\int_{M} d^{d+1} x \sqrt{g} R+\int_{\partial M} d^{d} x \sqrt{\gamma} 2 K\right],
$$

where $\kappa^{2}=8 \pi G_{d+1}, \gamma$ is the induced metric on $\partial M$ and $K$ is the trace of the extrinsic curvature of the boundary. This is the standard Einstein-Hilbert action with the Gibbons-Hawking boundary term which ensures that the variational problem is well-defined. The overall sign is chosen so that the action is positive definite when evaluated on a classical solution in the vicinity of (Euclidean) 
AdS. ${ }^{7}$ To allow for matter we add

$$
S_{m}=\int_{M} d^{d+1} x \sqrt{g} \mathcal{L}_{m}
$$

to the gravitational action, where $\mathcal{L}_{m}$ is a generic matter field Lagrangian density. The stress tensor is then defined in the standard fashion by

$$
\delta_{g} S_{m} \equiv \frac{1}{2} \int_{M} d^{d+1} x \sqrt{g} \tilde{T}_{\mu \nu} \delta g^{\mu \nu}
$$

The Euler-Lagrange equations of the total action $S=S_{g r}+S_{m}$ are Einstein's equations

$$
G_{\mu \nu}=\kappa^{2} \tilde{T}_{\mu \nu}
$$

and the matter field equations.

Our method of holographic renormalization makes use of the ADM formalism and the GaussCodacci equations which we will now briefly review. The standard ADM formalism (see, for instance, 23]) for a pseudo-Riemannian manifold relies on the existence of a global time function $t$ which is used to foliate space-time into diffeomorphic hypersurfaces of constant $t$. For a generic Riemannian manifold, however, there is no natural choice of time as all coordinates are equivalent. Nevertheless, for a Riemannian manifold with boundary one can use the coordinate 'normal' to the boundary as a global 'time' coordinate and, hence, foliate the manifold into hypersurfaces diffeomorphic to the boundary. For asymptotically (Euclidean) AdS manifolds this can always be done at least in a neighborhood of the boundary [12] (see also the recent review [14] and references therein). The question of if and where this 'radial' coordinate emanating from the boundary ceases to be well-defined depends on the topology of the space and will not be addressed here.

Let $r$ be the 'radial' coordinate emanating from the boundary of a Riemannian manifold with boundary $\left(M, g_{\mu \nu}\right)$ in the way described above and consider the hypersurfaces $\Sigma_{r}$ defined by $r(x)=$ constant. The unit normal to $\Sigma_{r}$, pointing in the direction of increasing $r$, is given by $n^{\mu}=$ $\left.\frac{1}{\|d r\|_{g}} g^{\mu \nu} \frac{\partial r}{\partial x^{\nu}}\right|_{\Sigma}$. This allows one to express the induced metric on the hypersurfaces in a coordinate independent fashion $\operatorname{as}^{8} \hat{\gamma}_{\mu \nu}=g_{\mu \nu}-n_{\mu} n_{\nu}$. The metric on $M$ can then be decomposed as

$$
d s^{2}=g_{\mu \nu} d x^{\mu} d x^{\nu}=\hat{\gamma}_{\mu \nu} d \hat{x}^{\mu} d \hat{x}^{\nu}+2 N_{\mu} d \hat{x}^{\mu} d r+\left(N^{2}+N_{\mu} N^{\mu}\right) d r^{2}
$$

where $N$ and $N^{\mu}$ are respectively the lapse function and the shift function. They correspond to nondynamical degrees of freedom which we will 'gauge-fix' shortly. Geometrically they measure how 'normal' the coordinate $r$ is to the hypersurfaces: the choice $N=1, N^{\mu}=0$ makes $r$ a Gaussian

\footnotetext{
${ }^{7}$ Our convention for the Riemann tensor is $R_{\rho \nu \sigma}^{\mu}=\partial_{\nu} \Gamma_{\rho \sigma}^{\mu}+\Gamma_{\lambda \nu}^{\mu} \Gamma_{\rho \sigma}^{\lambda}-(\nu \leftrightarrow \sigma)$. This differs by an overall sign from the conventions used in 6] 7].

${ }^{8}$ We use a hat to denote tensors that are purely transverse to the unit normal, i.e. quantities which vanish when contracted with $n^{\mu}$.
} 
normal coordinate, in which case $n^{\mu}$ becomes tangent to geodesics normal to the hypersurfaces. A quantity that will be of central importance in our analysis is the extrinsic curvature of the hypersurfaces

$$
\hat{K}_{\mu \nu}=\hat{\gamma}_{\mu}^{\rho} \nabla_{\rho} n_{\nu}=\frac{1}{2} £_{n} \hat{\gamma}_{\mu \nu},
$$

where $£_{n}$ denotes the Lie derivative with respect to the unit normal $n^{\mu}$. Thus, the extrinsic curvature measures the radial evolution of the induced metric and hence encapsulates all dynamical information of the geometry of the hypersurfaces. In fact, the Riemann tensor of the $\mathrm{d}+1 \mathrm{di}$ mensional manifold $M$ can be expressed entirely in terms of the intrinsic (i.e. Riemannian) and extrinsic curvatures of the hypersurfaces $\Sigma_{r}$ via the so called Gauss-Codacci equations

$$
\begin{aligned}
\hat{\gamma}_{\mu}^{\alpha} \hat{\gamma}_{\nu}^{\beta} \hat{\gamma}_{\rho}^{\gamma} \hat{\gamma}_{\sigma}^{\delta} R_{\alpha \beta \gamma \delta}= & \hat{R}_{\mu \nu \rho \sigma}+\hat{K}_{\mu \sigma} \hat{K}_{\nu \rho}-\hat{K}_{\mu \rho} \hat{K}_{\nu \sigma} \\
& \hat{\gamma}_{\nu}^{\rho} n^{\sigma} R_{\rho \sigma}=\hat{\nabla}_{\mu} \hat{K}_{\nu}^{\mu}-\hat{\nabla}_{\nu} \hat{K}_{\mu}^{\mu} .
\end{aligned}
$$

These purely geometric equations exhibit most explicitly the implications of the bulk $(d+1)$ dimensional geometry for the geometry of the hypersurfaces. For, instance, one sees immediately that conformal flatness of the bulk manifold implies very strong constraints on the extrinsic curvature of the radial slices. The case of interest to us here is, of course, the case of an Einstein bulk manifold. A little manipulation of the Gauss-Codacci equations brings them in the following form, most suitable to exhibit the consequences of $M$ being Einstein - we stress that the following equations are purely geometric:

$$
\begin{array}{r}
\hat{K}^{2}-\hat{K}_{\mu \nu} \hat{K}^{\mu \nu}=\hat{R}+2 G_{\mu \nu} n^{\mu} n^{\nu}, \\
\hat{\nabla}_{\mu} \hat{K}_{\nu}^{\mu}-\hat{\nabla}_{\nu} \hat{K}_{\mu}^{\mu}=G_{\rho \sigma} \hat{\gamma}_{\nu}^{\rho} n^{\sigma}, \\
£_{n} \hat{K}_{\mu \nu}+\hat{K}_{\mu \nu}-2 \hat{K}_{\mu}{ }^{\rho} \hat{K}_{\rho \nu}=\hat{R}_{\mu \nu}-\hat{\gamma}_{\mu}^{\rho} \hat{\gamma}_{\nu}^{\sigma} R_{\rho \sigma} .
\end{array}
$$

These equations become dynamical once we use Einstein's equations to replace the Einstein tensor with the matter stress tensor. When $M$ is both conformally flat and Einstein they can be solved exactly [24]. Note that conformal flatness is automatic if $M$ is three dimensional. However, solving these equations in general for an arbitrary Einstein manifold is equivalent to solving Einstein's equations and, therefore, far from trivial.

The ADM formalism allows us to express the bulk action in terms of transverse quantities as

$$
S=-\frac{1}{2 \kappa^{2}} \int_{M} d^{d+1} x \sqrt{\hat{\gamma}} N\left(\hat{R}+\hat{K}^{2}-\hat{K}_{\mu \nu} \hat{K}^{\mu \nu}-2 \kappa^{2} \mathcal{L}_{m}\right) .
$$


The canonical momenta can now be defined in the standard fashion ${ }^{9}$

$$
\pi^{\mu \nu} \equiv \frac{\delta L}{\delta \dot{\hat{\gamma}}_{\mu \nu}}=-\frac{1}{2 \kappa^{2}} \sqrt{\hat{\gamma}}\left(\hat{K} \hat{\gamma}^{\mu \nu}-\hat{K}^{\mu \nu}\right), \quad \pi^{I} \equiv \frac{\delta L}{\delta \dot{\Phi}_{I}},
$$

where $\Phi_{I}$ is a generic matter field and the Lagrangian $L$ is defined as usual by $S=\int d r L$. In particular, the canonical momenta conjugate to the lapse and shift functions vanish identically, and hence the corresponding equations of motion in the canonical formalism become constraints, which are precisely the first two equations in (39).

Let us finally consider the on-shell gravitational action, as it is precisely this quantity that becomes the generating functional of connected correlation functions of the dual field theory on the boundary. From Einstein's equation and (39) it follows that

$$
S_{\text {on-shell }}=-\frac{1}{\kappa^{2}} \int_{r=r_{0}}^{r=r_{1}} d r d^{d} x \sqrt{\hat{\gamma}} N\left[\hat{R}+\kappa^{2}\left(n^{\mu} n^{\nu} \tilde{T}_{\mu \nu}-\mathcal{L}_{m}\right)\right]
$$

where the boundary is located at $r=r_{1}$ and $r_{0}\left(r_{0}<r_{1}\right.$ by our definition of the unit normal $)$ defines a hypersurface in the interior of $M$. As mentioned above, there always exists an $r_{0}$ sufficiently close to $r_{1}$ such that the above expression for the on-shell action is well-defined, but there may not exist an $r_{0}$ such that the integration from $r_{0}$ to $r_{1}$ covers the entire manifold. However, this issue is irrelevant for the near boundary analysis. The on-shell action is a functional of the boundary values of the fields $\hat{\gamma}\left(r_{1}, x\right)$ and $\Phi_{I}\left(r_{1}, x\right)$. The corresponding momenta on $\Sigma_{r_{1}}$ are then obtained from the on-shell action by

$$
\pi^{\mu \nu}\left(r_{1}, x\right)=\frac{\delta S_{\text {on-shell }}}{\delta \hat{\gamma}_{\mu \nu}\left(r_{1}, x\right)}, \quad \pi^{I}\left(r_{1}, x\right)=\frac{\delta S_{\text {on-shell }}}{\delta \Phi_{I}\left(r_{1}, x\right)} .
$$

However, asymptotically AdS spaces are non-compact and the boundary is located at $r_{1}=\infty$. So the above expressions for the on-shell action and canonical momenta evaluated at $r_{1}$ contain divergences due to the infinite volume of the bulk manifold. The advantage of the Hamiltonian formulation is that these expressions hold identically for any hypersurface $\Sigma_{r}$ defined by any finite $r$.

This is then a good point to detail the philosophy of the new approach.

1. Using the Hamiltonian formalism we have arrived at a manifestly covariant expression for the canonical momenta evaluated on an arbitrary hypersurface $\Sigma_{r}$ - for finite $r$. In particular, the momenta are functionals of the bulk fields on $\Sigma_{r}$.

\footnotetext{
${ }^{9}$ One may consider adding extra finite local boundary terms in the action (32). These would result in additional terms in the momenta and finally lead to additional contact terms in (holographically computed) correlators. The addition of such boundary terms is the counterpart of finite local counterterms related to the scheme dependence of the boundary QFT.
} 
2. Using Einstein's equations in the Gauss-Codacci relations (39) - together with any extra equations of motion for matter fields - we obtain a set of second order differential equations for the induced metric and the other bulk fields evaluated on $\Sigma_{r}$.

3. This set of second order ordinary differential equations is then turned into a set of first order functional partial differential equations by expressing the radial derivative as a functional derivative. The crucial point here is that the canonical momenta are essentially the $r$-derivative of the corresponding bulk fields (up to issues relating to gauge fixing to be discussed below) and we have just seen that the momenta are functionals of the bulk fields on $\Sigma_{r}$. Hence,

$$
\frac{d}{d r}=\int d^{d} x 2 \hat{K}_{\mu \nu}[\hat{\gamma}, \Phi] \frac{\delta}{\delta \hat{\gamma}_{\mu \nu}}+\int d^{d} x \dot{\Phi}_{I}[\hat{\gamma}, \Phi] \frac{\delta}{\delta \Phi_{I}}
$$

This step is reminiscent, essentially equivalent, to the theorem of Jacobi [25] in the HamiltonJacobi theory of classical mechanics, where one expresses the momenta as functional derivatives of the on-shell action as above, but then derives a partial differential equation for the on-shell action. This is precisely the approach followed in [15]. However, we derive functional PDE's for the momenta and this is advantageous as we will discuss momentarily.

4. The set of first order functional PDE's thus obtained are, of course, much harder to solve than the original set of second order ODE's ${ }^{10}$, but this representation of the problem is most suitable for the near-boundary analysis in asymptotically AdS spaces, where the bulk fields satisfy prescribed but arbitrary Dirichlet boundary conditions:

$$
\hat{\gamma}_{\mu \nu} \sim \mathrm{e}^{2 r} \hat{\gamma}_{(0) \mu \nu}(x), \quad \Phi_{I} \sim \mathrm{e}^{\left(\Delta_{I}-d\right) r} \phi_{(0) I}(x)
$$

as $r \rightarrow \infty$, where $\Delta_{I}$ is the scaling dimension of the operator dual to the bulk field $\Phi_{I}$. Provided these asymptotics hold ${ }^{11}$, the asymptotic form of the functional representation of the radial derivative is very suggestive:

$$
\partial_{r} \sim \int d^{d} x 2 \hat{\gamma}_{\mu \nu} \frac{\delta}{\delta \hat{\gamma}_{\mu \nu}}+\int d^{d} x\left(\Delta_{I}-d\right) \Phi_{I} \frac{\delta}{\delta \Phi_{I}}
$$

Not surprisingly, this is the total dilatation operator, $\delta_{D}$, of the theory, which appears as a consequence of the well-defined scale transformation rules the fields obey asymptotically.

\footnotetext{
${ }^{10}$ In classical mechanics - where the PDE's are not functional - it is often easier to solve the PDE's (either for the action, i.e. the Hamilton-Jacobi equation, or for the momenta) and, as a result solve Hamilton's equations. This amounts to the 'inverse method of characteristics', as Hamilton's equations are just the characteristic equations for the Hamilton-Jacobi equation.

${ }^{11}$ When $\Delta=d / 2$ the leading asymptotics of the bulk fields are of the form $r \exp (-d r / 2)$. In those cases the functional representation of the radial derivative must be modified [26], but the above procedure for the asymptotic analysis applies equally well.
} 
From the point of view of the boundary field theory, this is precisely the Callan-Symanzik equation obeyed by the renormalized one-point functions in the presence of sources - which, as we will see, are related to the canonical momenta. In the spirit of perturbation theory then, it is natural to expand the momenta in eigenfunctions of the total dilatation operator and solve the functional PDE's 'perturbatively', i.e. asymptotically, while preserving covariance. This is in contrast to the method of holographic renormalization, where one is seeking asymptotic expansions of the bulk fields using the distance from the AdS boundary as a small parameter - thus explicitly breaking bulk covariance.

5. In contrast to previous methods, our focus here is on the canonical momenta and not the on-shell action. From the field theory point of view, this is to say we are interested in the exact one-point functions - as opposed to the partition function in the presence of sources. The connection between the canonical momenta and the one-point functions is surprisingly simple in our formalism. On the regulating surface $\Sigma_{r}$ with $r$ finite, the (unrenormalized but regulated) one-point functions are given by the AdS/CFT prescription as the functional derivative of the on-shell action with respect to the corresponding source, i.e. bulk field. Let us show this for the case of matter fields (the derivation for gravity is the same). The variation of the action is given by,

$$
\delta S=\left.\frac{\partial L}{\partial \dot{\Phi}_{I}} \delta \Phi_{I}\right|^{r}+\int^{r} d r \delta \Phi_{I}\left[\frac{\partial L}{\partial \Phi_{I}}-\partial_{r}\left(\frac{\partial L}{\partial \dot{\Phi}_{I}}\right)\right],
$$

where we assumed (without loss of generality) that the matter fields have a standard kinetic term and we gauge fixed as in (50). The second term is just the Euler-Lagrange equation and thus vanishes on-shell. We therefore obtain,

$$
\frac{\delta S_{\text {on-shell }}}{\delta \Phi_{I}}=\pi^{I}(r)
$$

where we used (411). The l.h.s. is, by the AdS/CFT dictionary, the regulated 1-point function in the presence of sources. We now argue that a similar connection holds for the renormalized one-point functions as well. The renormalized one-point functions are defined to be the one-point functions one obtains from the renormalized action. This is in turn the on-shell action plus a set of covariant counterterms which remove the divergences of the on-shell action as $r \rightarrow \infty$. Suppose now these covariant counterterms for the on-shell action are constructed. Taking the functional derivative with respect to the appropriate bulk field they lead to covariant terms which when added to the regularized momenta must - by construction - remove all the potential singularities from the canonical momenta. We thus need to identify the singular part of canonical momentum and remove it. The main constraint is that the subtraction should be covariant. This is done by expanding the momentum in terms of 
(covariant) eigenfunctions of the dilatation operator and observing that the divergent terms have eigenvalues less than the dimension $\Delta$ of the dual operator. To summarize, we have the very general result

$$
\left\langle\hat{T}_{\mu \nu}\right\rangle_{\mathrm{ren}}=-\frac{1}{\kappa^{2}}\left(\hat{K}_{(d) \mu \nu}-\hat{K}_{(d)} \hat{\gamma}_{\mu \nu}\right), \quad\left\langle\mathcal{O}_{I}\right\rangle_{\mathrm{ren}}=\frac{1}{\sqrt{\hat{\gamma}}} \pi_{\left(\Delta_{I}\right)}^{I}
$$

The terms on the right hand sides have the (engineering) dimension indicated by their subscript. These would have also been their dilatation eigenvalues in the absence of conformal anomalies and RG running. In most examples, the bulk theory involves non-trivially only fields of the gauged supergravity obtained by reducing the $10 \mathrm{~d}$ supergravity over a compact manifold. In such cases, the bulk fields correspond to operators of protected dimensions and thus the coefficients in (49) fail to be eigenfunctions of the dilatation operator only because of the conformal anomaly. As we shall see explicitly in the examples, the conformal anomaly induces an inhomogeneous term in the dilatation transformation of the coefficients in (49). We further note that these coefficients are not completely determined by the asymptotic analysis - they are the counterparts of the undetermined coefficients of the near-boundary analysis. It is therefore redundant to first construct covariant counterterms for the on-shell action and then use them to obtain the renormalized one-point functions, since the equations of motion can be solved for the momenta directly - and these are all one needs to obtain the one-point functions.

6. Although, as we just argued, it is not necessary to compute covariant counterterms for the on-shell action in order to obtain renormalized correlation functions, one can construct them as a secondary step in our method, and in fact more efficiently than previous methods. This is done by constructing a differential equation - essentially equivalent to the Hamilton-Jacobi equation - for the on-shell action which we then solve in parallel to the equations for the momenta. Explicit examples will be presented below.

In previous works where a Hamiltonian approach was used [15]-17] a central point of the analysis was the solution of the Hamilton-Jacobi (HJ) equation for the on-shell action. In this context, the HJ equation is a functional PDE for the on-shell action which can be solved by inserting an ansatz for the on-shell action in it. By requiring that terms with different number of derivatives cancel separately one gets a number of equations, the descent equations, that can be solved to determine the unknown functions in the ansatz. In the presence of scalars, the equations were further organized in [16] according to the number of scalar fields they contain. The resulting equations are not in general equivalent to the ones in the standard 
approach. This is due to the fact that the scalar fields are treated differently than in the standard holographic renormalization. Recall that in the standard approach the equations are solved by using the distance from the boundary as a small parameter with all sources being unconstrained. The expansion in the number of scalar fields requires that all scalar fields are (equally) small and for this to be the case the Dirichlet data (QFT sources) should be tuned to be (appropriately) small. This is rather unnatural since on the QFT side all sources are unconstrained and of order one and in general can lead to erroneous results. In simple examples, such as the ones studied in the literature, there is no obstruction in considering the sources small and the results so obtained are in agreement with results obtained via the standard method. An alternative approach that overcomes these issues is to organize the terms in the HJ equation according to their dilatation weight. This yields equations that are equivalent to the ones in the standard holographic renormalization method. Solving the HJ equation for the on-shell action leads to some of the same simplifications we find here. For instance, the covariant counterterm action is derived easier. On the other hand, the use of an ansatz for the on-shell action (instead of constructively obtaining the most general solution) as well as various sign ambiguities make the method less rigorous than the standard approach. More importantly, focusing on the canonical momenta instead of the on-shell action appears to be the most economic way to proceed.

7. Apart from an elegant framework for the general asymptotic analysis, this formalism provides a most efficient way to calculate correlation functions of the boundary field theory holographically. As we have just seen this amounts to determining the renormalized canonical momenta as functionals of arbitrary bulk fields - i.e. as functionals of arbitrary sources. To determine 2-point functions we only need to determine the momenta in terms of the source at linearized level. Furthermore, the contribution of the counterterms to 2-point functions can also be determined directly from the linearized analysis, following the discussion in the previous point. A similar discussion applies also to $n$-point functions $(n>2)$. This leads to a significant simplification of the computation of correlation functions. Details will appear elsewhere [26].

\section{Gauge fixing}

Before we carry out the near boundary analysis for pure AdS gravity and gravity coupled to scalars following the above prescription, let us fix the gauge freedom associated with the shift and lapse functions by setting $N^{\mu}=0$ and $N=1$. The bulk metric then takes the form ${ }^{12}$

$$
d s^{2}=d r^{2}+\gamma_{i j}(r, x) d x^{i} d x^{j}
$$

\footnotetext{
${ }^{12}$ All tensors are transverse and so we drop the hats form now on.
} 
where $i, j=1, \ldots d$ are indices along the hypersurfaces. The extrinsic curvature becomes

$$
K_{i j}=\frac{1}{2} \dot{\gamma}_{i j}
$$

where the dot denotes differentiation with respect to $r$. The non-vanishing components of the Christoffel symbol are

$$
\Gamma_{i j}^{d+1}=-K_{i j}, \quad \Gamma_{d+1 j}^{i}=K_{j}^{i}, \quad \Gamma_{j k}^{i} .
$$

The gravitational field equations (39) take the form

$$
\begin{array}{r}
K^{2}-K_{i j} K^{i j}=R+2 \kappa^{2} \tilde{T}_{d+1 d+1}, \\
\nabla_{i} K_{j}^{i}-\nabla_{j} K=\kappa^{2} \tilde{T}_{j d+1}, \\
\dot{K}_{j}^{i}+K K_{j}^{i}=R_{j}^{i}-\kappa^{2}\left(\tilde{T}_{j}^{i}+\frac{1}{1-d} \tilde{T}_{\sigma}^{\sigma} \delta_{j}^{i}\right) .
\end{array}
$$

$\dot{K}_{j}^{i}$ here stands for $\frac{d}{d r}\left(\gamma^{i k} K_{k j}\right)$. An additional equation for the on-shell action can be derived as promised. Since,

$$
\dot{S}_{\text {on-shell }}=L=-\frac{1}{\kappa^{2}} \int_{\Sigma_{r}} d^{d} x \sqrt{\gamma}\left[R+\kappa^{2}\left(\tilde{T}_{d+1 d+1}-\mathcal{L}_{m}\right)\right]
$$

we can obtain an expression for $S_{\text {on-shell }}$ if we write the integrand as the derivative of some (covariant) quantity. This is achieved by introducing a covariant variable $\lambda$ and writing

$$
S_{\text {on-shell }}=-\frac{1}{\kappa^{2}} \int_{\Sigma_{r}} d^{d} x \sqrt{\gamma}(K-\lambda) .
$$

Taking the trace of the third equation in (53) we determine that $\lambda$ satisfies

$$
\dot{\lambda}+K \lambda-\kappa^{2}\left(\mathcal{L}_{m}-\frac{1}{1-d} \tilde{T}_{\sigma}^{\sigma}\right)=0
$$

\subsection{Pure gravity case}

We will now demonstrate the method of Hamiltonian holographic renormalization for pure gravity with a negative cosmological constant ${ }^{13} \Lambda=d(1-d) / 2$. The equations of motion reduce to

$$
\begin{array}{r}
K^{2}-K_{i j} K^{i j}=R+d(d-1), \\
\nabla_{i} K_{j}^{i}-\nabla_{j} K=0, \\
\dot{K}_{j}^{i}+K K_{j}^{i}=R_{j}^{i}+d \delta_{j}^{i} .
\end{array}
$$

The on-shell action is determined from the equation

$$
\dot{\lambda}+K \lambda=d .
$$

\footnotetext{
${ }^{13} S \sim \int(R-2 \Lambda)$
} 
We will expand the extrinsic curvature and $\lambda$ in eigenfunctions of the dilatation operator, which now takes the form

$$
\delta_{D}=\int d^{d} x 2 \gamma_{i j} \frac{\delta}{\delta \gamma_{i j}}
$$

Then,

$$
\begin{array}{r}
K_{j}^{i}[\gamma]=K_{(0) j}^{i}+K_{(2) j}^{i}+\cdots+K_{(d) j}^{i}+\tilde{K}_{(d) j}^{i} \log \mathrm{e}^{-2 r}+\cdots, \\
\lambda[\gamma]=\lambda_{(0)}+\lambda_{(2)}+\cdots+\lambda_{(d)}+\tilde{\lambda}_{(d)} \log \mathrm{e}^{-2 r}+\cdots,
\end{array}
$$

where

$$
\begin{array}{r}
\delta_{D} K_{(n) j}^{i}=-n K_{(n) j}^{i}, n<d, \quad \delta_{D} \tilde{K}_{(d) j}^{i}=-d \tilde{K}_{(d) j}^{i}, \\
\delta_{D} K_{(d) j}^{i}=-d K_{(d) j}^{i}-2 \tilde{K}_{(d) j}^{i} \\
\delta_{D} \lambda_{(n)}=-n \lambda_{(n)}, \quad n<d, \quad \delta_{D} \tilde{\lambda}_{(d)}=-d \tilde{\lambda}_{(d)}, \\
\delta_{D} \lambda_{(d)}=-d \lambda_{(d)}-2 \tilde{\lambda}_{(d)} .
\end{array}
$$

The inhomogeneous transformations of $K_{(d)}{ }_{j}^{i}$ and $\lambda_{(d)}$, which follow immediately from the relation between the radial derivative and the dilatation operator, are due to the conformal anomaly.

Before we proceed to determine these coefficients from the equations of motion, let us exhibit the equivalence of this covariant expansion in eigenfunctions of the dilatation operator to the asymptotic expansion of the induced metric in the standard holographic renormalization method. There the induced metric is expanded in $\rho=\exp (-2 r)$ as

$$
\gamma_{i j}=\frac{1}{\rho}\left[g_{(0) i j}+\rho g_{(2) i j}+\cdots+\rho^{d / 2} g_{(d) i j}+\rho^{d / 2} \log \rho h_{(d) i j}+\cdots\right]
$$

Hence

$$
\frac{1}{2} \dot{\gamma}_{i j}=\frac{1}{\rho} g_{(0) i j}-\rho g_{(4) i j}+\cdots+\rho^{(d / 2-1)}\left[\left(1-\frac{d}{2}\right) g_{(d) i j}-h_{(d) i j}\right]+\rho^{(d / 2-1)} \log \rho\left(1-\frac{d}{2}\right) h_{(d) i j}+\cdots
$$

However, each term in the covariant expansion of the extrinsic curvature is a functional of the induced metric $\gamma_{i j}$. Using the expansion (62) of the metric we can functionally expand the eigenfunctions of the dilatation operator as

$$
\begin{array}{r}
K_{(0) i j}[\gamma]=\gamma_{i j}=\frac{1}{\rho}\left[g_{(0) i j}+\rho g_{(2) i j}+\cdots+\rho^{d / 2} g_{(d) i j}+\rho^{d / 2} \log \rho h_{(d) i j}+\cdots\right] \\
K_{(2) i j}[\gamma]=K_{(2) i j}\left[g_{(0)}\right]+\rho \int d^{d} x g_{(2) k l} \frac{\delta K_{(2) i j}}{\delta g_{(0) k l}}+\cdots \\
\vdots \\
K_{(d) i j}[\gamma]=\rho^{(d / 2-1)} K_{(d) i j}\left[g_{(0)}\right]+\cdots \\
\tilde{K}_{(d) i j}[\gamma]=\rho^{(d / 2-1)} \tilde{K}_{(d) i j}\left[g_{(0)}\right]+\cdots
\end{array}
$$


Inserting these expressions in the covariant expansion for $K_{i j}$ and comparing with (63) we determine

$$
\begin{array}{r}
K_{(0) i j}\left[g_{(0)}\right]=g_{(0) i j}, \\
K_{(2) i j}\left[g_{(0)}\right]=-g_{(2) i j}\left[g_{(0)}\right], \\
\vdots \\
K_{(n) i j}\left[g_{(0)}\right]=-\frac{n}{2} g_{(n) i j}\left[g_{(0)}\right]+\text { lower, } \\
\vdots \\
K_{(d) i j}\left[g_{(0)}\right]=-\frac{d}{2} g_{(d) i j}\left[g_{(0)}\right]-h_{(d) i j}\left[g_{(0)}\right]+\text { lower } \\
\tilde{K}_{(d) i j}\left[g_{(0)}\right]=-\frac{d}{2} h_{(d) i j}\left[g_{(0)}\right],
\end{array}
$$

where 'lower' stands for terms involving functional derivatives with respect to $g_{(0) i j}$ of lower order coefficients $g_{(k) i j}\left[g_{(0)}\right]$. For $\mathrm{d}=4$, for example,

$$
K_{(4) i j}\left[g_{(0)}\right]=-2 g_{(4) i j}\left[g_{(0)}\right]-h_{(4) i j}\left[g_{(0)}\right]+\int d^{4} x g_{(2) k l} \frac{\delta g_{(2) i j}\left[g_{(0)}\right]}{\delta g_{(0) k l}} .
$$

Therefore, there is a one-to-one correspondence between the terms in the asymptotic expansion of holographic renormalization and our covariant expansion in eigenfunctions of the dilatation operator. In particular, the non-local terms in the two expansions are related, whereas the coefficients of the logarithms - which are related to the conformal anomaly - are just proportional to each other. This completes our demonstration of the equivalence of the two methods.

The new formulation, however, is advantageous over the standard method in that the onshell action is expressed entirely in terms of the extrinsic curvature coefficients, for arbitrary $d$. Furthermore, the one-point function in the presence of sources is also expressed simply in terms of one of the extrinsic curvature coefficients. The asymptotic analysis is done once, for all $d$, resulting in generic recursion relations for the extrinsic curvature coefficients.

To complete the near boundary analysis one then just needs to solve the recursion relations for a given dimension $d$. The key ingredient in our method which allows for these improvements is the functional relation between the canonical momenta and the on-shell action, namely

$$
\pi^{i j}=-\frac{1}{2 \kappa^{2}} \sqrt{\gamma}\left(K \gamma^{i j}-K^{i j}\right)=\frac{\delta S_{\text {on-shell }}}{\delta \gamma_{i j}},
$$

or

$$
K \gamma^{i j}-K^{i j}=\frac{2}{\sqrt{\gamma}} \frac{\delta}{\delta \gamma_{i j}} \int_{\Sigma_{r}} d^{d} x \sqrt{\gamma}(K-\lambda) .
$$

Inserting the covariant expansions for $K_{j}^{i}$ and $\lambda$ we can relate the coefficients of the on-shell action 
to those of the extrinsic curvature as

$$
\begin{array}{r}
K_{(2 n) j}^{i}=\lambda_{(2 n)} \delta_{j}^{i}-\frac{2}{\sqrt{\gamma}} \int d^{d} x \sqrt{\gamma} \gamma_{k j} \frac{\delta}{\delta \gamma_{i k}}\left(K_{(2 n)}-\lambda_{(2 n)}\right), 0 \leq n \leq \frac{d}{2} \\
\tilde{K}_{(d) j}^{i}=\tilde{\lambda}_{(d)} \delta_{j}^{i}-\frac{2}{\sqrt{\gamma}} \int d^{d} x \sqrt{\gamma} \gamma_{k j} \frac{\delta}{\delta \gamma_{i k}}\left(\tilde{K}_{(d)}-\tilde{\lambda}_{(d)}\right) .
\end{array}
$$

The trace of these equations then gives

$$
\left(1+\delta_{D}\right) K_{(2 n)}=\left(d+\delta_{D}\right) \lambda_{(2 n)}, \quad 0 \leq n \leq \frac{d}{2}, \quad\left(1+\delta_{D}\right) \tilde{K}_{(d)}=\left(d+\delta_{D}\right) \tilde{\lambda}_{(d)} .
$$

Since we know how the coefficients transform under the dilatation operator, these relations completely determine $\lambda$ in terms of the trace of the extrinsic curvature. Namely we obtain the significant result

$$
\lambda_{(2 n)}=\frac{(2 n-1)}{(2 n-d)} K_{(2 n)}, 0 \leq n \leq \frac{d}{2}-1, \quad \tilde{\lambda}_{(d)}=\frac{d-1}{2} K_{(d)}, \quad \tilde{K}_{(d)}=0 .
$$

The coefficient $K_{(2 n) j}^{i}$ are only determined for $n<d / 2$. If one does the computation for general $d$ then the corresponding expression has a first order pole at $d=2 n$. A short computation using (69) shows that the residue of the pole is exactly $\tilde{K}_{(d) j}^{i}$, i.e. the coefficient of the logarithmic term in $d$ dimensions,

$$
\tilde{K}_{(d) j}^{i}=\lim _{n \rightarrow d / 2}\left(\left(n-\frac{d}{2}\right) K_{(2 n) j} \underset{i}{i}\right) .
$$

In practice one can also use this result in order to compute $K_{(d-2) j}^{i}$ in $d$ dimensions from $\tilde{K}_{(d-2)}{ }_{j}$ in $d-2$ dimensions.

We thus arrive at a general closed form expression for the covariant counterterm action that renders the on-shell action finite:

$$
S_{c t}=\frac{(1-d)}{\kappa^{2}} \int_{\rho=\epsilon} d^{d} x \sqrt{\gamma}\left[\sum_{m=0}^{\frac{d}{2}-1} \frac{1}{(2 m-d)} K_{(2 m)}+\frac{1}{2} K_{(d)} \log \epsilon\right]
$$

The rest of the analysis is now straightforward. First, by direct substitution of the covariant expansion of the extrinsic curvature into the first equation in (57) one finds a recursive relation for the traces of the extrinsic curvature coefficients, namely

$$
\begin{gathered}
K_{(2)}=\frac{R}{2(d-1)}, \\
K_{(2 n)}=\frac{1}{2(d-1)} \sum_{m=1}^{n-1}\left[K_{(2 m) i j} K_{(2 n-2 m)} i j-K_{(2 m)} K_{(2 n-2 m)}\right], \quad 2 \leq n \leq \frac{d}{2}
\end{gathered}
$$


Finally, inserting the values of $\lambda_{(2 n)}$ and the traces of the extrinsic curvature we have determined in (71) and (74) into the functional relation (69) one can evaluate all coefficients recursively. In doing so, one sees that considerable simplifications occur upon using the second equation in (57), which implies

$$
\nabla_{i} K_{(2 n) j}^{i}-\nabla_{j} K_{(2 n)}=0, \quad 0 \leq n \leq \frac{d}{2}, \quad \nabla_{i} \tilde{K}_{(d) j}^{i}-\nabla_{j} \tilde{K}_{(d)}=0 .
$$

Note that although $K_{(d) j}^{i}$ is non-local in general, its trace is local as follows from (74). Carrying out the above procedure is straightforward but the result becomes of forbidding complexity as one goes up in dimension. The algorithm, however, could be implemented in a computer code which would in principle calculate the counterterms and the holographic Weyl anomaly for any dimension. For illustrative purposes we quote the results for up to four dimensions,

$$
\begin{gathered}
\mathbf{d}=\mathbf{2} \\
K_{j}^{i}[\gamma]=\delta_{j}^{i}+K_{(2) j}^{i}+\ldots \\
K[\gamma]=d+P+\ldots \\
S_{\mathrm{ct}}=\frac{(d-1)}{\kappa^{2}} \int_{\rho=\epsilon} d^{2} x \sqrt{\gamma}\left[1-\frac{1}{4} R \log \epsilon\right] \\
\mathbf{d = 4} K_{j}^{i}[\gamma]=\delta_{j}^{i}+P_{j}^{i}+\frac{1}{2}\left[\frac{1}{2}\left(P^{k l} P_{k l}-P^{2}\right) \delta_{j}^{i}\right. \\
\left.-\frac{1}{(d-2)}\left(2 R^{i}{ }_{k j l} P^{k l}-P R_{j}^{i}+\square P_{j}^{i}-\nabla^{i} \nabla_{j} P\right)\right] \log \epsilon+K_{(4) j}^{i}+\ldots \\
K[\gamma]=d+P+\frac{1}{2(d-1)}\left(P^{k l} P_{k l}-P^{2}\right)+\ldots \\
S_{\mathrm{ct}}=\frac{(d-1)}{\kappa^{2}} \int_{\rho=\epsilon} d^{4} x \sqrt{\gamma}\left[1+\frac{1}{(d-2)} P-\frac{1}{4(d-1)}\left(P^{k l} P_{k l}-P^{2}\right) \log \epsilon\right]
\end{gathered}
$$

where we have introduced the sectional curvature tensor

$$
P_{i j}=\frac{1}{(d-2)}\left(R_{i j}-\frac{1}{2(d-1)} R \gamma_{i j}\right)
$$

which transforms under Weyl rescalings of the metric $\delta \gamma_{i j}=-2 \gamma_{i j} \delta \sigma$ as $\delta P_{i j}=\nabla_{i} \nabla_{j} \delta \sigma$. 


\subsection{Gravity coupled to scalars}

Having carried out in detail the near boundary analysis for pure AdS gravity in our formalism, we will now briefly describe how the analysis can be generalized to include scalars. In this case the matter action takes the form

$$
S_{m}=\int_{M} d^{d+1} x \sqrt{g}\left[\frac{1}{2} g^{\mu \nu} \partial_{\mu} \Phi_{I} \partial_{\nu} \Phi_{I}+V\left(\Phi_{I}\right)\right] .
$$

Along with the gravitational field equations (53) and equation (56) for the on-shell action, we now have the equations of motion for the scalar fields

$$
\ddot{\Phi}_{I}+K \dot{\Phi}_{I}+\square \Phi_{I}-\frac{\partial}{\partial \Phi_{I}} V(\Phi)=0 .
$$

In terms of the canonical momenta ${ }^{14} \pi^{I}=\dot{\Phi}_{I}$,

$$
\dot{\pi}^{I}+K \pi^{I}+\square \Phi_{I}-\frac{\partial}{\partial \Phi_{I}} V(\Phi)=0
$$

Next, we expand the canonical momenta and on-shell action in eigenfunctions of the dilatation operator

$$
\delta_{D}=\int d^{d} x 2 \gamma_{i j} \frac{\delta}{\delta \gamma_{i j}}+\int d^{d} x\left(\Delta_{I}-d\right) \Phi_{I} \frac{\delta}{\delta \Phi_{I}} .
$$

In addition to the expansions (60) we now have expansions for the canonical momenta of the scalar fields

$$
\pi^{I}[\gamma, \Phi]=\sum_{d-\Delta_{I} \leq s<\Delta_{I}} \pi_{(s)}^{I}+\pi_{\left(\Delta_{I}\right)}^{I}+\tilde{\pi}_{\left(\Delta_{I}\right)}^{I} \log \mathrm{e}^{-2 r}+\cdots
$$

The crucial difference is that the momenta now depend on all bulk fields and not just the induced metric. Moreover, depending on the scaling dimension $\Delta_{I}$, the eigenvalues of the dilatation operator may not be integers anymore. The analysis is exactly analogous to that for pure AdS gravity, making essential use of the functional relations (68) and

$$
\pi^{I}=-\frac{1}{\kappa^{2}} \frac{1}{\sqrt{\gamma}} \frac{\delta}{\delta \Phi_{I}} \int d^{d} x \sqrt{\gamma}(K-\lambda)
$$

which imply the key relation

$$
\left(1+\delta_{D}\right) K+\kappa^{2}\left(\Delta_{I}-d\right) \pi^{I} \Phi_{I}=\left(d+\delta_{D}\right) \lambda
$$

As for pure AdS gravity, this can be used to express the coefficients of $\lambda$ in terms of those of the momenta. Then, inserting $\lambda$ into (68) and (82), the canonical momenta are determined iteratively.

\footnotetext{
${ }^{14}$ Strictly speaking, the momenta are densities and should include a factor of $\sqrt{\gamma}$ as we defined them earlier, see for instance 23. Nevertheless, we will drop (with due care) this factor in this section as this results in simpler equations.
} 
As an illustration, consider the case of two scalar fields, $\Phi$ and $\Sigma$, both of scaling dimension $\Delta=3$ in $d=4$ and with potential that has a critical point at $\Phi=\Sigma=0$. The most general potential compatible with these requirements is

$$
V(\Phi, \Sigma)=\sum_{n=0}^{\infty} \sum_{m=0}^{n} \kappa^{n-2} V_{(m, n-m)} \Phi^{m} \Sigma^{n-m} .
$$

where $V_{(0,0)}=\Lambda / \kappa^{2}$ is the cosmological constant, $V_{(0,1)}=V_{(1,0)}=0$, i.e. there are no linear couplings, $V_{(1,1)}=0$ and $V_{(2,0)}=V_{(0,2)}=-3$, i.e. the quadratic terms are diagonal in $\Phi$ and $\Sigma$ and both have mass $m^{2}=\Delta(\Delta-d)=-3$, and all other coupling $V_{(m, m-n)}$ are arbitrary. The iterative approach determines the following on-shell action:

$$
\begin{gathered}
S_{\text {on-shell }}=-\frac{1}{\kappa^{2}} \int_{\rho=\epsilon} d^{4} x \sqrt{\gamma}\left\{\frac{d-1}{d-2} P-\frac{1}{4}\left[P_{i j} P^{i j}-P^{2}-\kappa^{2} \Phi(\square+P) \Phi\right.\right. \\
\left.\left.-\kappa^{2} \Sigma(\square+P) \Sigma\right] \log \epsilon+K_{(4)}-\lambda_{(4)}+\ldots\right\}-\int_{\rho=\epsilon} d^{4} x \sqrt{\gamma} W(\Phi, \Sigma)
\end{gathered}
$$

where the "superpotential" is given by

$$
\begin{gathered}
W(\Phi, \Sigma)=\frac{1}{\kappa^{2}}(d-1)+\frac{1}{2}\left(\Phi^{2}+\Sigma^{2}\right)+ \\
\frac{\kappa}{d-3}\left(V_{(3,0)} \Phi^{3}+V_{(2,1)} \Phi^{2} \Sigma+V_{(1,2)} \Phi \Sigma^{2}+V_{(0,3)} \Sigma^{3}\right)+ \\
\kappa^{2}\left[\left(\frac{1}{2} V_{(4,0)}-\frac{1}{4(d-3)^{2}}\left(9 V_{(3,0)}^{2}+V_{(1,2)}^{2}\right)+\frac{d}{16(d-1)}\right) \Phi^{4}+\right. \\
\left(\frac{1}{2} V_{(3,1)}-\frac{1}{(d-3)^{2}}\left(3 V_{(3,0)} V_{(2,1)}+V_{(1,2)} V_{(0,3)}\right)\right) \Phi^{3} \Sigma+ \\
\left(\frac{1}{2} V_{(2,2)}-\frac{1}{2(d-3)^{2}}\left(3 V_{(3,0)} V_{(1,2)}+\right.\right. \\
\left.\left.3 V_{(0,3)} V_{(2,1)}+2 V_{(2,1)}^{2}+2 V_{(1,2)}^{2}\right)+\frac{d}{8(d-1)}\right) \Phi^{2} \Sigma^{2}+ \\
\left(\frac{1}{2} V_{(0,4)}-\frac{1}{4(d-3)^{2}}\left(9 V_{(0,3)}^{2}+V_{(2,1)}^{2}\right)+\frac{d}{16(d-1)}\right) \Sigma^{4}+ \\
\left.\left(\frac{1}{2} V_{(1,3)}-\frac{1}{(d-3)^{2}}\left(3 V_{(0,3)} V_{(1,2)}+V_{(2,1)} V_{(3,0)}\right)\right) \Phi \Sigma^{3}\right] \log \epsilon+W_{(4)}+\ldots
\end{gathered}
$$

A direct computation shows that $W$ satisfies

$$
V\left(\Phi_{I}\right)=\frac{1}{2}\left[\left(\frac{\partial W}{\partial \Phi_{I}}\right)^{2}-\frac{d \kappa^{2}}{d-1} W^{2}\right] .
$$

and thus can be considered as a "superpotential". The AdS critical point of $V$ is also a critical point of $W$. This together with (87) guarantee gravitational stability of the AdS critical point 
and of BPS domain-wall solutions of the gauged supergravity [27, 28]. The expression (87) can be derived in general in this formalism, i.e. for arbitrary dimension and scalar fields [15. In this context one may also view (87) as a differential equation that should be solved to determine the "superpotential". Since (87) is quadratic in $W$ it can only determine $W$ up to a sign. In contrast, our method guarantees that the "superpotential" satisfies (87), but nevertheless there is no sign ambiguity in the determination of (86).

So far we have presented a method to solve the first order functional differential equations asymptotically. It would be, of course, desirable to solve these non-linear equations completely for arbitrary sources, but this is beyond present capabilities. However, we can get an idea of the difficulty of the problem by considering the easiest part, namely the superpotential for one scalar field. The full superpotential can be determined (up to sign that is fixed using the asymptotic solution for $W$ ) by equation (87) which is a first order ODE, as opposed to the first order functional differential equations that determine the rest of the on-shell action. To solve for the superpotential we observe that it is possible to bring (87) into the form of Abel's equation [29]:

$$
y^{\prime}(\psi)=\frac{v^{\prime}}{v} y^{3}-y^{2}-\frac{v^{\prime}}{v} y+1
$$

where $\psi=\sqrt{\frac{d \kappa^{2}}{d-1}} \phi, y=\operatorname{coth}(u), W=v \cosh (u)$, and $v$ is related to the potential by $\frac{2(d-1)}{d \kappa^{2}} V=-v^{2}$. The general solution to this equation is not known, but it can be solved in special cases. For example, the potential

$$
V(\psi)=-\frac{d(d-1)}{2 \kappa^{2}} \cosh \left(\frac{2}{3} \psi\right)
$$

leads to a soluble equation, with solution

$$
W(\psi)=\frac{(d-1) \cosh ^{1 / 2}\left(\frac{2}{3} \psi\right)\left(\cosh \left(\frac{2}{3} \psi\right)+\cosh ^{1 / 2}(\gamma) \operatorname{sech}^{1 / 2}\left(\frac{4}{3} \psi+\gamma\right)\right)}{\kappa^{2} \sqrt{1+\cosh (\gamma) \operatorname{sech}\left(\frac{4}{3} \psi+\gamma\right)+2 \cosh \left(\frac{2}{3} \psi\right) \cosh ^{1 / 2}(\gamma) \operatorname{sech}^{1 / 2}\left(\frac{4}{3} \psi+\gamma\right)}}
$$

Here $\gamma$ is an arbitrary parameter, and the scaling dimension, $\Delta$, of the operator dual to the scalar field is $2 d / 3$. Both the potential and the superpotential have $\phi=0$ as a critical point. $W$ has an expansion around zero

$$
W(\psi)=\frac{d-1}{\kappa^{2}}\left(1+\frac{1}{6} \psi^{2}+\frac{1}{27} \tanh (\gamma) \psi^{3}+\ldots\right)
$$

Since $\Delta=2 d / 3$ the term cubic in $\phi$ has a dilatation eigenvalue $3(\Delta-d)=-d$ which is exactly the correct order where the asymptotic expansion of the on-shell action breaks down and an undetermined term arises. In this case it happens that there is no logarithmic term, but a new parameter $\gamma$ appears at the correct order. 


\section{Conclusions}

We reviewed in this paper how QFT data is encoded in geometry via the AdS/CFT correspondence and we developed a new, more powerful, calculational method. A central element in the extraction of the QFT data is the form of asymptotic solutions for Einstein and matter field equations. The asymptotic solutions contain, after a number of terms that are uniquely determined in terms of the Dirichlet data, a term that is only partially determined by the asymptotic analysis. At the same order a logarithmic term may appear. We can now summarize the way the QFT data are encoded as follows:

- The undetermined coefficient encodes all correlation functions. To uncover them one needs exact solutions with arbitrary Dirichlet data.

- The relations that the undetermined coefficients satisfy are related to QFT Ward identities, including anomalies.

- The logarithmic term is also related to the conformal anomaly.

The conformal anomaly appears also as a coefficient of the logarithmic divergence of the on-shell action [6]. The relation between logarithmic divergences in the on-shell action and the logarithmic term in the asymptotic expansion of a (free) scalar field has also recently appeared in the math literature [30, 31] in studies related to the so-called Q-curvature ${ }^{15}$. It would be interesting to understand the significance of the Q-curvature in terms of the dual quantum field theory. Let us also emphasise again that in terms of physics it is most important to obtain information about the undetermined coefficients by studying, for instance, the constraints implied by regularity in the interior.

We have presented a new method for obtaining renormalized correlation functions, conformal anomalies and Ward identities of the boundary QFT from geometrical data. The method is a Hamiltonian version of the standard approach but with the radial coordinate playing the role of time. In this approach the renormalized one-point functions of the boundary QFT in the presence of sources are related to the canonical momenta of the bulk fields. The near-boundary expansions of the standard method are replaced by covariant expansions in eigenfunctions of the dilatation operator. This leads to simple closed form expressions for the counterterms and for the renormalized one-point functions in terms of the coefficients of the covariant expansion of the momenta, valid in all dimensions. For the coefficients we derived general recursion relations (also valid in any dimension). This leads to a more efficient algorithm for determining counterterms and one-point

\footnotetext{
${ }^{15}$ The Q-curvature is a generalization of the scalar curvature in two dimensions: it satisfies analogous conformal transformation properties.
} 
functions than in previous works. It would be important to integrate the recursion relation in general. It seems likely that this would require a more intuitive and geometric understanding of the conformal anomaly.

\section{Acknowledgments}

We would like to thank W. Mück for comments on the first version of this work. KS is supported by NWO.

\section{References}

[1] J. M. Maldacena, "The large $N$ limit of superconformal field theories and supergravity," Adv. Theor. Math. Phys. 2, 231 (1998) [Int. J. Theor. Phys. 38, 1113 (1999)] arXiv:hep-th/9711200.

[2] S. S. Gubser, I. R. Klebanov and A. M. Polyakov, "Gauge theory correlators from non-critical string theory," Phys. Lett. B 428, 105 (1998) arXiv:hep-th/9802109.

[3] E. Witten, "Anti-de Sitter space and holography," Adv. Theor. Math. Phys. 2, 253 (1998) arXiv:hep-th/9802150.

[4] O. Aharony, S. S. Gubser, J. M. Maldacena, H. Ooguri and Y. Oz, "Large N field theories, string theory and gravity," Phys. Rept. 323 (2000) 183 arXiv:hep-th/9905111.

[5] E. D'Hoker and D. Z. Freedman, "Supersymmetric gauge theories and the AdS/CFT correspondence," arXiv:hep-th/0201253.

[6] M. Henningson and K. Skenderis, "The holographic Weyl anomaly," JHEP 9807 (1998) 023 arXiv:hep-th/9806087; M. Henningson and K. Skenderis, "Holography and the Weyl anomaly," Fortsch. Phys. 48 (2000) 125 arXiv:hep-th/9812032.

[7] S. de Haro, S. N. Solodukhin and K. Skenderis, "Holographic reconstruction of spacetime and renormalization in the AdS/CFT correspondence," Commun. Math. Phys. 217 (2001) 595 arXiv:hep-th/0002230.

[8] M. Bianchi, D. Z. Freedman and K. Skenderis, "How to go with an RG flow," JHEP 0108 (2001) 041 arXiv:hep-th/0105276; "Holographic renormalization," Nucl. Phys. B 631 (2002) 159 arXiv:hep-th/0112119.

[9] K. Skenderis, "Lecture notes on holographic renormalization," Class. Quant. Grav. 19 (2002) 5849 arXiv:hep-th/0209067. 
[10] V. Balasubramanian and P. Kraus, "A stress tensor for anti-de Sitter gravity," Commun. Math. Phys. 208, 413 (1999) arXiv:hep-th/9902121.

[11] P. Kraus, F. Larsen and R. Siebelink, "The gravitational action in asymptotically AdS and flat spacetimes," Nucl. Phys. B 563 (1999) 259 arXiv:hep-th/9906127.

[12] C. Fefferman and C. Robin Graham, "Conformal Invariants", in Elie Cartan et les Mathématiques d'aujourd'hui (Astérisque, 1985) 95.

[13] C.R. Graham, "Volume and Area Renormalizations for Conformally Compact Einstein Metrics", math.DG/9909042.

[14] M.T. Anderson, "Geometric Aspects of the AdS/CFT correspondence", hep-th/0403087.

[15] J. de Boer, E. Verlinde and H. Verlinde, "On the holographic renormalization group," JHEP 0008 (2000) 003 arXiv:hep-th/9912012 ; J. de Boer, "The holographic renormalization group," Fortsch. Phys. 49, 339 (2001) arXiv:hep-th/0101026.

[16] D. Martelli and W. Muck, "Holographic renormalization and Ward identities with the Hamilton-Jacobi method," Nucl. Phys. B 654 (2003) 248 arXiv:hep-th/0205061.

[17] M. Fukuma, S. Matsuura and T. Sakai, "A note on the Weyl anomaly in the holographic renormalization group," Prog. Theor. Phys. 104, 1089 (2000) arXiv:hep-th/0007062; J. Kalkkinen and D. Martelli, "Holographic renormalization group with fermions and form fields," Nucl. Phys. B 596, 415 (2001) arXiv:hep-th/0007234; J. Kalkkinen, D. Martelli and W. Muck, "Holographic renormalisation and anomalies," JHEP 0104, 036 (2001) arXiv:hep-th/0103111; M. Fukuma, S. Matsuura and T. Sakai, "Holographic renormalization group," Prog. Theor. Phys. 109, 489 (2003) arXiv:hep-th/0212314.

[18] A. Petkou and K. Skenderis, "A non-renormalization theorem for conformal anomalies," Nucl. Phys. B 561 (1999) 100 arXiv:hep-th/9906030.

[19] H. Osborn and A. C. Petkou, "Implications Of Conformal Invariance In Field Theories For General Dimensions," Annals Phys. 231 (1994) 311 arXiv:hep-th/9307010.

[20] L. Bonora, P. Pasti and M. Bregola, "Weyl Cocycles," Class. Quant. Grav. 3 (1986) 635.

[21] S. Deser and A. Schwimmer, "Geometric Classification Of Conformal Anomalies In Arbitrary Dimensions," Phys. Lett. B 309 (1993) 279 arXiv:hep-th/9302047. 
[22] M. Bianchi and A. Marchetti, "Holographic three-point functions: One step beyond the tradition," arXiv:hep-th/0302019 M. Bianchi, M. Prisco and W. Muck, "New results on holographic three-point functions," JHEP 0311 (2003) 052 arXiv:hep-th/0310129; W. Muck and M. Prisco, arXiv:hep-th/0402068.

[23] R.M. Wald, General Relativity, The University of Chicago Press, 1984.

[24] K. Skenderis and S. N. Solodukhin, "Quantum effective action from the AdS/CFT correspondence," Phys. Lett. B 472 (2000) 316 arXiv:hep-th/9910023.

[25] R. Courant and D. Hilbert, Methods of Mathematical Physics, Vol. II, Interscience Publishers, 1962.

[26] I. Papadimitriou and K. Skenderis, in preparation.

[27] P. K. Townsend, "Positive Energy And The Scalar Potential In Higher Dimensional (Super)Gravity Theories," Phys. Lett. B 148, 55 (1984).

[28] K. Skenderis and P. K. Townsend, "Gravitational stability and renormalization-group flow," Phys. Lett. B 468, 46 (1999) arXiv:hep-th/9909070.

[29] Dr. E. Kamke, Differentialgleichungen Lösungsmethoden und Lösungen, Chelsea Publishing Company, 1971.

[30] C.R. Graham and M. Zworski, "Scattering matrix in conformal geometry", math.DG/0109089

[31] C. Fefferman and C.R. Graham, "Q-curvature and Poincare metrics", math.DG/0110271. 